\title{
Whole genome analysis of clinical Staphylococcus aureus multi-drug resistant isolates from Moscow medical center
}

\author{
Yulia Mikhaylova \\ Central Research Institute \\ of Epidemiology \\ Moscow, Russia \\ mihailova@cmd.su \\ Valeria Fomina \\ National Medical and Surgical Center \\ named after N.I. Pirogov \\ Moscow, Russia \\ med_2006@mail.ru
}

\author{
Andrey Shelenkov \\ Central Research Institute \\ of Epidemiology \\ Moscow, Russia \\ 0000-0002-7409-077X
}

\author{
Mikhail Zamyatin \\ National Medical and Surgical Center \\ named after N.I. Pirogov \\ Moscow, Russia \\ mnz1@yandex.ru \\ Vasiliy Akimkin \\ Central Research Institute \\ of Epidemiology \\ Moscow, Russia \\ vgakimkin@yandex.ru
}

\author{
Yurii Yanushevich \\ Central Research Institute \\ of Epidemiology \\ Moscow, Russia \\ yanushevich@cmd.su \\ Dmitry Shagin \\ Central Research Institute \\ of Epidemiology \\ Moscow, Russia \\ shagin@cmd.su
}

\begin{abstract}
Staphylococcus aureus is a Gram-positive bacterium that is a usual member of human body microbiota and can be frequently found in the upper respiratory tract and on the skin. It can become an opportunistic pathogen causing skin infections including abscesses, respiratory tract infections and food poisoning. Methicillin-resistant strains of $S$. aureus represent one of the major sources of healthcare-associated infections in clinical settings. Although more than 10,000 $S$. aureus genomes are currently available in public databases, the lack of associated metadata usually does not allow conducting the studies on antibiotic resistance acquisition, epidemiological surveillance and the presence of virulent factors in this important pathogen. We have performed whole-genome sequencing (WGS) of 22 clinical $\boldsymbol{S}$. aureus isolates on Illumina and Oxford Nanopore platforms. Isolate typing was performed using Multilocus sequence typing (MLST), antimicrobial resistance factors were revealed using both phenotypical and genomic studies, virulence factors and plasmids were annotated in genome sequences. These data will be useful for future epidemiological and antimicrobial resistance studies of S. aureus.
\end{abstract}

Keywords - NGS, pathogenic bacteria, whole genome sequencing, antibiotic resistance

\section{Introduction}

A number of factors determine the role of Staphylococcus aureus as both commensal and pathogenic bacteria. These include many determinants of virulence, as well as the ability to acquire multiple resistance to antimicrobial drugs. Infections caused by methicillin-resistant $S$. aureus (MRSA) have become a global problem.

The aim of this work is to perform whole genome sequencing (WGS)-based genotyping and genomic epidemiology analysis of multi-drug resistant $S$. aureus isolates including MRSA. In addition, several isolates obtained from the same patient were sequenced to investigate the optimal SNP threshold value for distinguishing close and distantly related isolates by their core genome comparison.
Materials and methods

WGS was performed on Illumina HiSeq platform for 22 isolates of $S$. aureus including two environmental isolates collected from Moscow medical center, and two isolates were also sequenced on MinION platform (Oxford Nanopore). Genomic DNA was isolated with DNeasy Blood and Tissue kit (Qiagen) and used for paired-end library preparation with Nextera $^{\text {TM }}$ DNA Sample Prep Kit (Illumina $\left.{ }^{\circledR}\right)$. For MinION platform, DNA was used to prepare the MinION library with the Rapid Barcoding Sequencing kit SQK-RBK004 and was sequenced on R9 SpotON flow cell. The susceptibility was determined by the boundary concentration method on VITEK2Compact30 analyzer (bioMerieux, France).

Genome assemblies were made using SPAdes version 3.13 [1] and Unicycler [2] (hybrid long- and short read assemblies). Genome comparisons were performed using roary [3], dnadiff and custom software. Custom annotation pipeline was used for MLST and spa-typing, antibiotic resistance gene prediction, virulence factor and plasmid search using corresponding versions of public databases.

$$
\text { results }
$$

The population of $S$. aureus studied was represented by two dominating lines (ST8 and ST22) and 5 minor lines including one isolate possessing a novel sequence type (ST5555) and having previously unknown MLST allele (pta_647). The isolates studied were divided into 11 Spa variants with prevailing Spa-type t008. Seventeen MRSA isolates had SCCmec IV type, and one - SCCmec V cassette.

Other common determinants of antibiotic resistance were genes of resistance to penicillin, macrolides and amikacin. The set of virulence factors in $S$. aureus isolates was quite extensive (up to 20 genes), and included both structural components of the cell and secreted products. Pairwise comparisons of core-genome composition and the number of single nucleotide polymorphisms (SNP) of isolates from the same and different patients, revealed discrepancies in number of SNPs and thresholds previously proposed for strain discrimination in literature. For example, the threshold of 15 SNPs for distinguishing $\mathrm{S}$. aureus isolates was proposed 
earlier [4], while we have revealed up to 51 nucleotide mismatches between the core genomes of our isolates from the same patient.

\section{Conclusions}

Thus, applying WGS technology enabled us to reveal unique genome features of $S$. aureus clinical isolates. Such features are important both from the standpoint of an increased level of virulence of MRSA and evolutionary changes. The data obtained will allow us to identify new target molecules for differentiation of MRSA isolates belonging to different genetic variants. Our results suggest that although the number of core-genome SNPs proves to be useful measure for distinguishing distant bacterial strains, more research is needed to establish appropriate thresholds for it.

\section{REFERENCES}

[1] Bankevich A, Nurk S, Antipov D, Gurevich AA, Dvorkin M, Kulikov AS, et al. SPAdes: a new genome assembly algorithm and its applications to single-cell sequencing. J Comput Biol. 2012;19(5):45577

[2] Wick RR, Judd LM, Gorrie CL, Holt KE. Unicycler: Resolving bacterial genome assemblies from short and long sequencing reads. PLoS Comput Biol. 2017;13(6):e1005595

[3] Page AJ, Cummins CA, Hunt M, Wong VK, Reuter S, Holden MT, et al. Roary: rapid large-scale prokaryote pan genome analysis. Bioinformatics. 2015;31(22):3691-3

[4] Schurch et al. (2018) Clinical Microbiology and Infection, 24:350-354 